\title{
Artículos
}

\section{POLÍTICA DE EDUCACIÓN SEXULA EN PUERTO RICO: EL ANÁLISIS DE TRES VERSIONES DEUNA POLÍTICA PÚBLICA EN DECADENCIA}

\author{
Irvyn E. Nieves-Rolón"
}

\section{Resumen}

La participación del Estado en la educación sexual de la niñez y adolescencia se postula a través de las Políticas de educación sexual. En la última década se han implementado tres políticas de educación sexual en Puerto Rico. Estas tres políticas denominadas "Política Pública de Educación en Salud Sexual en las Escuelas Públicas del Departamento de Educación" han sido implantadas en los años 2004, 2011 y 2012. Este artículo discute las tres políticas de educación sexual. En la medida que una política suplanta la otra, se hacen más evidentes las tendencias conservadoras del Estado. Al comparar las políticas, se observa retroceso en la atención de las circunstancias y necesidades de formación sexual que enfrentan la niñez y adolescencia de Puerto Rico. En la discusión presentada se hace evidente los cambios que ha enfrentado la política de educación sexual. Asimismo, se elabora la reflexión sobre sus implicaciones.

[Descriptores: política educativa, educación sexual, política de educación sexual, política comparada].

\section{Abstract}

State involvement in the sexual education of children and adolescents is postulated through sexual education policies. In the last decade three sexual education policies were implemented in Puerto Rico. These policies are called "Department of Education Public Schools' Sexual Health Education Public Policy" and were

\footnotetext{
Catedrático Auxiliar, Departamento de Trabajo Social, Escuela de Ciencias Sociales y Humanas, Universidad del Este, Puerto Rico.
} 
implemented in the years 2004, 2011 and 2012. This article discusses these three sexual education policies. While a new policy overrides the other, it's becoming more evident the implications of a conservative State. When comparing these policies, it is observed the declining attention to the sexual education needs of children and adolescents in Puerto Rico.

[Keywords: education policy, sexual education, sexual education policy, comparative policy].

\section{Introducción}

Las políticas educativas, atienden administrativa y políticamente un sin fin de aspectos complejos en relación a la gestión educativa de un país. No se trata únicamente de determinar el contenido curricular de cursos a ofrecer, sino que deben considerar la comprensión de procesos estructurales, pedagógicos y de desarrollo humano; conflictos institucionales; discusión e integración de diferentes posturas colectivas; y gestionar diversas acciones o intervenciones (Rivas, 2004). Las políticas educativas deben procurar proactividad para el logro de metas, efectividad en el logro de las mismas y conciliación entre los sectores que confligen o son atendidos por la política.

Las políticas públicas de educación sexual, por su parte, son las posturas del Estado para atender la formación en salud sexual del estudiantado, primordialmente niños, niñas y adolescentes. Aunque en el 1999 se implantó la primera política educativa sobre la educación en salud sexual, los alcances de la misma fueron escasos y quedan fuera del análisis realizado en este artículo que se concentra en los esfuerzos realizados en la última década. En este periodo, el Departamento de Educación de Puerto Rico ha implantado, mediante Cartas circulares, tres políticas de educación sexual. Estas tres políticas llevan el mismo nombre, específicamente "Política Pública de Educación en Salud Sexual en las Escuelas Públicas del Departamento de Educación" (Departamento de Educación, 2004, 2011, 2012). En la medida que una política es derogada para establecer una nueva, es de esperarse que la última sea más eficiente que la anterior para atender las circunstancias del estudiantado. La evidencia revisada en este análisis nos distancia de esa presunción. 


\section{La Política Pública de Educación en Salud Sexual en las Escuelas Públicas: versión 2004}

La primera de las tres políticas comparadas en este artículo, se implantó en el año 2004. Ésta estableció 11 áreas de contenido, a saber: (a) Fortalecer el Programa de Salud escolar; (b) Desarrollar un plan estratégico a corto y largo plazo para ofrecer programas de educación sexual integral desde el nivel elemental hasta el superior; (c) Crear un equipo interagencial e interdisciplinario para hacer un esfuerzo colaborativo y amplio para promover la salud sexual; (d) Crear un equipo de personas de la comunidad para enfrentar el impacto de los medios de comunicación; (e) Recopilar estudios para identificar el comportamiento sexual de riesgo; (f) Ofrecer programas para la formación de padres, madres o encargados; (g) Ampliar y fortalecer los programas de horario extendido con actividades supervisadas; (h) Adiestrar al personal docente y al profesional de apoyo del Programa de salud escolar sobre la inteligencia emocional; (i) Utilizar los medios de comunicación, tales como la televisión, la radio, la red cibernética y el teatro para apoyar los esfuerzos educativos del salón de clases; (j) Evaluar las estrategias de servicios y promover los ofrecimientos a grupos con necesidades particulares; (k) Revisar y armonizar el currículo con nuevos cambios en el sistema legal relacionados con aspectos de la sexualidad (Departamento de Educación, 2004). Todas estas áreas son discutidas en el texto de la política y demuestran amplitud e integración metodológica, administrativa y de contenido.

En un estudio realizado anteriormente analicé esta política en detalle. Entre las conclusiones de dicho estudio se encontraba que "aunque dicha política tiene aspectos que hay que mejorar -particularmente en el área de atención de las circunstancias de género- ésta satisface muchas de las necesidades que enfrentan los y las adolescentes en Puerto Rico" (Nieves-Rolón, 2010, p.150). Los aspectos señalados a mejorar, según presenta el estudio, se centraron en que aunque la política reconocía la necesidad de que se considere el género en la educación sexual, no propone medidas específicas que reconozcan que las circunstancias que forman a chicos y chicas son diferentes y con implicaciones distintas para cada cual. Otra crítica a esa política es que la misma fue pobremente implementada. Ni siquiera los propios maestros de salud quienes se suponían la implementaran, 
conocían la misma (Nieves-Rolón, 2010). Esto trae a colación que las políticas pueden redactarse con particular adecuación, pero, si no se invierten esfuerzos concertados al implementarla, pierde sentido el esfuerzo realizado.

\section{La Política Pública de Educación en Salud Sexual en las Escuelas Públicas: versión 2011}

Al comparar la política implementada en el 2004 con la implementada en el 2011 se observan considerables similitudes que llevan a cuestionarse, en primera instancia, el propósito de invertir esfuerzos y recursos para derogar la primera. Por ejemplo, en ambas políticas se encuentran postulados sobre: el respeto y la dignidad de la niñez y adolescencia; el proceso educativo como ente formativo y que guía a la prevención; una alegada visión integral de la sexualidad; integración y colaboración interagencial y comunitaria en favor de la educación sexual; capacitación de profesionales; incluir a padres y madres en los procesos relacionados a educación sexual; y la influencia de los medios de comunicación en la educación sexual y conductas de niños, niñas y adolescentes.

No obstante, al mirar en detalle ambas políticas se evidencia que la segunda carece de especificidad, y contrario a la primera, evita hacer planteamientos concretos de contenido temático para su implementación. Esto queda evidenciado al observarse que la Política del 2004 planteaba aspectos específicos a discutir con el estudiantado de acuerdo con su nivel escolar. La Política del 2011 hace planteamientos genéricos sobre lo que se debe discutir sin considerar que las necesidades del estudiantado varían drásticamente de acuerdo a su nivel escolar.

Debe señalarse que, la política del 2004 presentaba su fundamento teórico discutiendo aspectos como: la relevancia de la etapa de desarrollo de la persona adolescente en relación a su capacidad para establecer relaciones de compromiso; datos estadísticos que describen conductas y situaciones de los adolescentes; discusión sobre la educación sexual y sus metas; y la familia como educadora sexual primaria. A diferencia de lo anterior, la Política del 2011 contiene tres oraciones de fundamentos teóricos y una presentación de un diagrama basado en el libro: Sexualidad: Sus conceptos básicos de Mock y Martínez (1995). El diagrama citado de Mock y Martínez (1995) presenta aspectos muy relevantes, como el papel del género, 
el comportamiento erótico, la orientación sexual y los estilos de vida sexual. Sin embargo el texto de la política carece de una explicación alusiva o fundamentada en el diagrama. Es decir no se establece ni infiere una conexión entre la base teórica y los postulados que establece la política.

El único aspecto en el que considero que la Política del 2011 supera la del 2004, es que en su última cláusula hace explícita la necesidad de que se evalúe la implementación de la misma, aspecto que no se encuentra presente en la Política del 2004. Este señalamiento resulta de alguna manera paradójico en la medida que se propone la evaluación de efectividad pero no las guías específicas para su implementación. Vale mencionar que por regla general, el Estado no tiene cultura de evaluación de la implementación de sus políticas y representa una iniciativa saludable que se incluya en el texto de la política el propósito de evaluarla (Bardach, 2000).

\section{La Política Pública de Educación en Salud Sexual en las Escuelas Públicas: versión 2012}

Continuando con la comparación, es necesario destacar que la política del 2011 fue implantada -y a su vez derogadaantes de cumplir nueve meses de establecida. La misma se firmó el 19 de julio de 2011 y la política que le sustituyó se impuso el 2 de abril de 2012. Los medios noticiosos del país, informaron que el cambio en la política se debió a presiones del sector religioso del país y a reclamos para que se eliminaran los planteamientos relacionados a la perspectiva de género y a que se revisara cualquier referencia que pudiera conducir a "la enseñanza indiscriminada del 'sexo seguro' entre los jóvenes" (RodríguezAndino, 2011).

Al comparar ambas políticas, 2011 y 2012 , se observa que la mayor parte del texto permanece igual. No obstante, hay unos cambios puntuales y significativos entre una política y la otra. A continuación presento una descripción de los cambios entre la política del 2011 y la del 2012.

Observando las políticas, página por página, se ven las primeras diferencias en la página 3 , en la sección de Fundamentos teóricos. Las tres oraciones y el diagrama planteados como fundamentos teóricos en la política del 2011, fueron sustituidos por 5 párrafos elaborados, incluyendo citas de referencias bibliográficas. No obstante, cuando se lee su contenido se observa que desaparecen palabras como género, 
comportamiento erótico y orientación sexual que se encontraban en el diagrama de la política del 2011. Esta acción responde a una gestión del Estado apoderada por una ideología conservadora. Con esta ideología se utiliza al Estado para, alegadamente, resguardar los valores tradicionales de la sociedad e imponer criterios de corte moralistas y religiosos. Siendo así, una política que surge de este tipo de Estado, no puede considerar o incluir aspectos que resultan ser tabúes $u$ objetos de control, como la orientación sexual o el comportamiento erótico.

Incidentalmente, los cambios realizados representan un retroceso más en la política pública sobre educación sexual, desde una perspectiva de equidad y justicia social. Es decir, que un esfuerzo político por la educación sexual que ya había sido degradado, se convierte ahora en un esfuerzo más conservador y ortodoxo.

El próximo cambio se encuentra en el cuarto planteamiento de la sección Aspectos relevantes. Este planteamiento hace referencia a datos estadísticos de la Encuesta de Conductas de Riesgo en Adolescentes (YRBSS, por sus siglas en inglés). Sin embargo, en esta ocasión no queda clara la motivación para la realización de los cambios, además de ser obvio que en alguna de las dos versiones hay errores, involuntarios o intencionados, en la interpretación del dato estadístico. Por ejemplo, ambas políticas hacen referencia al mismo estudio. Sin embargo, la política del 2011 dice que de seis (6) conductas de riesgo, tres (3) han manifestado aumento en los últimos años mientras que, la política del 2012 plantea que de cuatro (4) conductas de riesgo, dos (2) han manifestado aumento. Otra discrepancia, en el mismo párrafo, es que la primera plantea que hubo un aumento en el uso del condón, a un $41.8 \%$ y la segunda plantea una disminución en el uso del condón en un $19.6 \%$.

En el sexto planteamiento de los Aspectos relevantes, la política del 2011 establece una serie de criterios sobre la salud sexual integral. El criterio (d) lee: "cultivar relaciones con personas de ambos sexos de forma equitativa..." (Departamento de Educación, 2011, p.5) y fue cambiada en la política del 2012 por "cultivar relaciones de amistad..." (Departamento de Educación, 2012, p.5). Las razones para este cambio no quedan claras, pero podría inferir que la redacción de la primera pudiese abrir las puertas para aceptar relaciones homosexuales o a tratar a los hombres y mujeres equitativamente. Sin embargo, ello no sería 
cónsono con la ideología conservadora de la administración gubernamental que impuso dicha política y representaría un conflicto ideológico para la misma. Asimismo, la política sustituye un aspecto de equidad en las relaciones de género por plantear la amistad como alternativa. Aunque, en esas edades puede ser muy saludable fomentar relaciones de amistad, ello no debe sustituir la equidad entre las relaciones. De esta forma, hay que reconocer la contradicción inherente del Estado que implanta una política para tratar la educación sexual de adolescentes, evadiendo los temas de género y prácticas sexuales.

Otros cambios se observan en las premisas ( $f, g, h)$ del sexto planteamiento de esta sección. La premisa (f) modifica una cláusula que planteaba "posponer la actividad sexual o ser capaz de asumir responsabilidad..." (2011, p.5), mientras la nueva política le añade "lograr cultivar una relación estable de compromiso y fidelidad mutua" (2012, pp. 5-6). De esta forma, se excluye la atención a aquellos sectores de la sociedad que no procuran o interesan responder a la idea de matrimonio, monógama o familia burguesa, favorecida por los discursos moralistas. Entiéndase, que no se provee apertura para el derecho, motivaciones o validez que tienen los individuos para ceñirse a los discursos de su preferencia, ya sean de índole conservador o liberal. No obstante, es necesario resaltar que lo que se propone en el documento es una política pública, no un código de conducta de alguna entidad privada o religiosa. Por tanto, es indispensable atender las diversidades de preferencias y conductas sostenidas en la sociedad desde una perspectiva educativa y salubrista.

En la política del 2012, la cláusula (g) acerca de la protección sexual fue modificada. Esta versaba sobre la protección si se está activo sexualmente, pero eliminaron una introducción a esa premisa que leía "proteger su salud sexual integral" (2011, p.5). También, la política del 2011 incluía una premisa (h) que leía "respetar la diversidad sexual y los derechos de las personas a tener su propia concepción de los patrones de expresión de la identidad sexual". La nueva política, eliminó dicha premisa. La nueva política no incluye en su lenguaje el respeto hacia la diversidad y por ende no se promueve una visión de aceptar las diferencias.

Ante circunstancias en las cuales las ideologías conservadoras se han apoderado del Estado, las posturas del 
gobierno prefieren no reconocer la diversidad sexual y expresar lo contrario resultaría ser una afrenta contra la moral que dichos sectores defienden. Ante ello es relevante considerar, que quienes dirigen los esfuerzos del Estado, con estas gestiones ganan legitimidad ante instituciones de mucho arraigo en la sociedad -como las religiosas-, quienes corresponderán apoyado la permanencia de éstos en el poder.

Un séptimo planteamiento en la sección de Aspectos relevantes fue eliminado. La Política del 2011 -que lo incluíaincorporaba posturas acerca de la actividad sexual de los adolescentes. Del trasfondo anterior que permea en el lenguaje de la Política, queda claro que en la última versión se prefiere evadir o mantener limitada la discusión relevante a la actividad sexual, de forma que se cubra mínimamente las preocupaciones del ala salubrista del Estado sin exacerbar los ánimos del sector conservador del país. Resulta sumamente cuestionable e irónico que una política de educación sexual evada hablar sobre sexo.

Una próxima premisa de esta sección, plantea aspectos argumentos como 'neurociencia' y justifica que los adolescentes no están capacitados para manejar los impulsos sexuales y la toma de decisiones responsables. Aun así, en la Política del 2012 le añadieron una oración para insistir en la importancia de que se fomente el dominio propio. Por un lado, concurro en la importancia de que se discuta con los adolescentes aspectos como autocontrol, manejo de presión grupal y otros temas afines. No obstante, debo cuestionar el énfasis en tratar estos temas de forma atada a la implantación y asimilación de discursos conservadores y represivos en vez de dirigir al estudiantado hacia el desarrollo de la capacidad crítica.

En otra sección de la política, se plantea el Modelo de educación para la salud sexual integral (p.6). Entre los postulados en que se basa dicho modelo, la Política del 2011 plantea el respeto a la dignidad de la niñez y adolescencia y su derecho de obtener información correcta sobre sexualidad según su nivel educativo. Pero, la Política del 2012 añadió que esto debe hacerse "reconociendo la necesidad de contar con la opinión, aprobación e integración de los padres o encargados" $(2012$, p.7). De forma que, para que se provea información sobre asuntos sexuales habrá que tener el endoso de sus padres.

Otras dos adiciones en esta sección de la política tratan el tema de la abstinencia y la relación de pareja. En uno de 
los puntos se añade la abstinencia que en la política del 2011 se presentaba como "promover la posposición de la actividad sexual" (2011, p.7). Además, en otro punto se añade una nueva premisa que lee: "promover una relación estable de compromiso y fidelidad mutua" (2012, p.7). Planteamientos como estos en la versión de la Política 2012 son alusivos a la visión de la familia burguesa, conservadora y de grupos moralistas.

La abstinencia sexual es un derecho que tiene cada persona, y por supuesto, cada adolescente. El problema es que, ese derecho es el más que procura atenderse y en ese ejercicio se niega otro derecho, el de ser informado sobre el sexo y las prácticas sexuales (Nieves-Rolón, 2010). Con la abstinencia que defiende la política, la actividad sexual será pospuesta hasta que se cumpla con otro criterio de control que corresponde a sostener una relación "estable de compromiso y fidelidad mutua". Tal como señala Foucault (1998), para conservar el poder de una clase dominante, el Estado institucionaliza definiciones atadas a dogmatismos religiosos y las defiende justificándolas como tradición.

La premisa (d) de esta sección en la Política del 2012, modifica la premisa (c) de la política del 2011. Esta premisa versa sobre los estudiantes que están activos sexualmente y las modificaciones realizadas giran en torno a intervenir con ellos discutiendo muchos aspectos desde retomar la abstinencia hasta métodos para prevenir embarazos y contagios, pero "todo ello reconociendo la necesidad de contar con la opinión, aprobación e integración de los padres o encargados" (2012, p.8). Una vez más se enfatiza sobre este particular. Concurro en que es necesario procurar la participación a padres, madres y encargados. No obstante, es preciso reconocer que dicho ejercicio debe realizarse buscando apoyar la toma de responsabilidad por parte de los progenitores, pero no como un mecanismo para que el Estado evada su responsabilidad con la salud sexual y la educación pública.

En la premisa (f) de la sección en discusión, se modificó el lenguaje que estipulaba "equidad entre los géneros" para estipular "dignidad del hombre y la mujer". Se reniega la utilización del concepto género, para implantar el concepto subjetivo y manipulable "dignidad". Asimismo, eliminaron una premisa que leía "se promoverá el respeto hacia las personas que tengan una orientación sexual diferente" (2011, p.8). Una vez más se 
evidencia que la política vigente evade explícitamente respetar a las personas que no respondan a las definiciones hegemónicas de la sexualidad. Es necesario entender esta acción en el contexto de un gobierno conservador, aliado a sectores que rechazan la homosexualidad y las prácticas sexuales fuera de relaciones legitimadas por el propio Estado o la Iglesia. En esta misma dirección, insertaron en la política una premisa sobre respeto a la dignidad humana que se basa en la Constitución de Puerto Rico que, por supuesto, no menciona ni el género ni la preferencia sexual.

En el séptimo postulado del Modelo de educación implantado, se eliminó una premisa que planteaba que se tratara el tema de "el papel del género y la sexualidad" en la capacitación que se ofrecerá a personal del Departamento de Educación. Los últimos cambios en la política van dirigidos a insistir en la integración de los padres y a incluir el programa "Tus valores cuentan" en la educación sexual que se ofrece en la escuela.

Una de las manifestaciones más obvias observadas en los cambios ocurridos entre las tres políticas estudiadas, es el trato que se le hace a la consideración del género en la formación y educación del estudiantado. Entre la Política del 2004 y la Política del 2011 se observa una disminución en el trato del género y entre la Política del 2011 y el 2012 se observa renuencia a tan siquiera mencionarlo.

Las razones para este antagonismo, no quedan claras. No obstante, en el año 2008 el Departamento de Educación implementó la "Política Pública sobre la Incorporación de la perspectiva de Género en la Educación Pública Puertorriqueña (Departamento de Educación, 2008). La misma fue implementada en el 2008 y derogada en el 2009 como una de las primeras gestiones de la Administración que entró en funciones en ese momento. La razón para dejar sin efecto a la política respondió a las presiones de grupos conservadores y a que, incluso, fue parte de las promesas de campaña política del partido que asumió el poder (Mercado, 2011).

Luego de realizar este análisis, debo resumir enfatizando algunos puntos. Es imprescindible que se reconozca el respeto a la diversidad y que los procesos educativos del Estado sirvan a tal propósito. Propongo la creación de grupos de diálogo que asesoren los procesos educativos para que puedan atenderse las preocupaciones de diversos sectores, pero cuidando la función 
del Estado. Es necesario respetar el derecho de los sectores moralistas a defender la educación que desean para sus hijos e hijas, pero sin pretender generalizar a manera de Política Pública creencias particulares. Esto, principalmente cuando los resultados de investigación indican la necesidad de continuar educando abiertamente sobre salud sexual. Las implicaciones de no hacerlo, acarrean altos costos para la sociedad en sus diversos componentes.

El sector religioso puede continuar valiéndose de sus iglesias y la educación privada para adoctrinar. Asimismo, estos sectores deben reconocer que en la función del Estado debe primar el bienestar colectivo y los esfuerzos que convienen a la salud del Pueblo. Por consiguiente, tanto las personas hacedoras de política pública, como quienes tienen poder político y administrativo en el Departamento de Educación deben tener claras sus responsabilidades para no convertir las políticas educativas en manuales de doctrina religiosa.

Las gestiones del Estado no pueden quedar sujetas a la influencia única de grupos particulares. Deben procurarse acciones conciliadoras por parte del Estado y gestiones proactivas que provean espacio para creencias diversas. Sin embargo, la responsabilidad educativa y salubrista del Estado tiene que prevalecer en su gestión. 


\section{Referencias}

Bardach, Eugine. (2000) A practical guide for policy analysis: The eightfold path to more effective problem solving. New York: Seven Bridges Press.

Departamento de Educación. (2004). Política Pública de Educación en Salud Sexual en las Escuelas Públicas del Departamento de Educación. San Juan: Estado Libre Asociado de Puerto Rico.

Departamento de Educación. (2008). Política Pública sobre la incorporación de la perspectiva de género en la educación pública puertorriqueña. San Juan: Estado Libre Asociado de Puerto Rico.

Departamento de Educación. (2011). Política Pública de Educación en Salud Sexual en las Escuelas Públicas del Departamento de Educación. San Juan: Gobierno de Puerto Rico.

Departamento de Educación. (2012). Política Pública de Educación en Salud Sexual en las Escuelas Públicas del Departamento de Educación. San Juan: Gobierno de Puerto Rico.

Foucault, Michael. (1998). Historia de la sexualidad 1: La voluntad del saber. México: Siglo veintiuno editores, s.a de c.v.

Mercado, Marta A. (2011). La perspectiva de género en la educación: Un análisis con método mixto de las políticas educativas de equidad de género en Puerto Rico. (Disertación Doctoral No Publicada). Universidad de Puerto Rico, Recinto de Río Piedras, Escuela Graduada de Trabajo Social.

Mock, Gloria, \& Martínez, Wilfred. (1995). Sexualidad: Sus conceptos básicos. San Juan: Editorial Cultural.

Nieves-Rolón, Irvyn E. (2010). La Política Pública de Educación en Salud Sexual en Puerto Rico: Implementación, Proceso Educativo y Visión Sobre los Géneros. Cuaderno de Investigación en la Educación, 25. Facultad de Educación de la Universidad de Puerto Rico, Recinto de Río Piedras.

Nieves-Rolón, Irvyn E. (2012). El varón adolescente y su educación sexual: Consonancias y disonancias entre la Política pública de educación en salud sexual en las escuelas públicas del Departamento de Educación y la socialización sexual de los varones adolescentes. (Disertación Doctoral No Publicada). Universidad de Puerto Rico, Recinto de Río Piedras, Escuela Graduada de Trabajo Social.

Rivas, José Ignacio. (2004). Política educativa y prácticas pedagógicas. BARBECHO: Revista de Reflexión Socioeducativa. Recuperado de http://www.barbecho.uma.es/DocumentosPDF/ BARBECHO4/A7B4.pdf

Rodríguez-Andino, Arys L. (2011, 9 de agosto). Obispo reclama otro currículo. Primera Hora. Recuperado de http://www.primerahora. com/obisporeclamaotrocurriculo-533603.html 